\title{
Oscillation criteria for a class of second order neutral delay differential equations
}

\author{
Yanxiang Shi*, Di Liu \\ School of Mathematical Sciences, Shanxi University, Taiyuan, China
}

Email address:

hongyu@sxu.edu.cn (Yanxiang Shi), liudi@sxu.edu.cn (Di Liu)

\section{To cite this article:}

Yanxiang Shi, Di Liu. Oscillation Criteria for a Class of Second Order Neutral Delay Differential Equations. Pure and Applied Mathematics Journal. Vol. 3, No. 5, 2014, pp. 95-98. doi: 10.11648/j.pamj.20140305.11

\begin{abstract}
Oscillation criteria for a class of second order neutral delay differential equations of the form $[c(t)((x(t)+$ $\left.p t x(t-\tau))^{\prime}\right) \alpha^{\prime}+q t f x t-\sigma=0, t \geq t 0$ is studied. By using first and second mean value theorem of integrals, the new sufficient condition is obtained and the corresponding result what was already obtained is generalized by the result in this paper.
\end{abstract}

Keywords: Neutral Delay Differential Equation, Oscillatory Solution, Eventually Positive Solution

\section{Introduction}

In this paper, we are concerned with the oscillation behavior for the second order neutral delay differential equations of the form

$$
\left[c(t)\left((x(t)+p(t) x(t-\tau))^{\prime}\right)^{\alpha}\right]^{\prime}+q(t) f(x(t-\sigma))=0, t \geq t_{0} .
$$

We assume that

(1) $c(t) \in C\left(\left[t_{0}, \infty\right), R\right), c(t)>0, \int_{t_{0}}^{\infty} \frac{d t}{\frac{1}{c}(t)}=\infty$;

(2) $\alpha \geq 1, \tau$ and $\sigma$ are nonnegative constants;

(3) $q(t) \in C\left(\left[t_{0}, \infty\right), R\right), q(t)>0$ and not identically equal to 0 on $\left[t^{*}, \infty\right), t^{*} \geq t_{0}$;

(4) $p(t) \in C\left(\left[t_{0}, \infty\right), R\right)$ and $0 \leq p(t) \leq 1$;

(5) $f(x) \in C(R, R)$ and $x f(x)>0, x \neq 0$.

Neutral differential equations have a fairly wide range of applications in the study of natural phenomena, and many natural phenomena can be modeled by neutral differential equations. Neutral differential equations with delay are the common mathematical model described in a large number of phenomena and processes, common in electronics, physics and other aspects. For example: delay differential equations are used to describe the dynamic system with feedback. This research have received considerable attention, and caused extensive interest. The mathematical analysis of neutral delay differential equations is often used for assessment of the properties of the solution due to the time delay, and the sufficient conditions for oscillatory of these equations.

Second order neutral delay differential equations evolved on the basis of the first order, and the oscillation sufficient conditions are obtained by applying the methods similar to an order but more advanced, and get the model to be promoted. The interval oscillation, linear oscillation, and oscillation of second-order nonlinear neutral differential equations are studied in recent years, which promote delay differential equations to a new level, and many scholars propose some new oscillation criteria. The oscillation of neutral differential equations are studied in [1], [5], [6] and [8], and the oscillation of neutral delay differential equations are studied in [2]-[4], [7], [9] and [10]. In this paper, we introduced neutral delay differential equations, with reference to [4] and [9]. The second order neutral delay differential equations of the form

$$
\left[r(t)\left((x(t)+p(t) x(t-\tau))^{\prime}\right)^{\alpha}\right]^{\prime}+f(t, x(t-\sigma))=0, t \geq t_{0}
$$

is studied in [4] and the equation is oscillatory if

$$
\underset{t \rightarrow \infty}{\limsup } \int_{t_{0}}^{t}\left(\Phi(s)-\frac{r(s-\sigma)}{(1+\alpha)^{(1+\alpha)}}\right) F^{1+\alpha}(t, s) \exp \left(\int_{t_{0}}^{s} F(t, \varepsilon) d \varepsilon\right) d s=+\infty \text {. }
$$

The second order neutral delay differential equations of the form

$$
\left[a(t)(x(t)+p(t) x(t-\tau))^{\prime}\right]^{\prime}+q(t) f(x(t-\sigma))=0, t \geq t_{0}
$$


is studied in [9] and obtained the new oscillation condition

$$
\limsup _{t \rightarrow \infty} \frac{1}{H\left(t, t_{0}\right)} \int_{t_{0}}^{t} H(t, s) \rho(s) q(s) f(1-p(s-\sigma)) d s=\infty .
$$

In this paper, these two literatures play an important role in the selection of the equation and the proof of the theorem.

The paper is organized as follows. In the next section, we present preliminaries including two definitions and three lemmas used in proof of the main results. In Section 3, we carry out the theorem for oscillatory of equation and detailed proof. Finally, some conclusions are given in Section 4.

\section{Preliminaries}

In order to prove the theorem we shall need the following definitions and lemmas.

Definition 1. A nontrivial solution $x(t)$ of Eq. (1) is called oscillatory in $\left[t_{0}, \infty\right), t_{0}>0$ if it has arbitrarily large zeros.

Otherwise the solution is called non-oscillatory.

Definition 2. Neutral differential Eq. (1) is called be oscillatory if all its solutions are oscillatory.

Lemma 3. Suppose that $x(t)$ is an eventually positive solution of Eq. (1), let

$$
z(t)=x(t)+p(t) x(t-\tau)
$$

then there exists a number $T_{0} \geq t_{0}+\tau+\sigma$, such that

$$
\left[c(t)\left(z^{\prime}(t)\right)^{\alpha}\right]^{\prime} \leq 0, z^{\prime}(t)>0, z(t)>0, z(t)>x(t), t \geq T_{0} .
$$

Proof. Since $x(t)$ is an eventually positive solution of Eq. (1), there exists a number $T_{0} \geq t_{0}+\tau+\sigma$, such that

$$
x(t)>0, x(t-\tau)>0, x(t-\tau-\sigma)>0, t \geq T_{0}, T_{0} \geq t_{0}+\tau+\sigma .
$$

From (2), we have $z(t)>0, z(t)>x(t)$, and

$$
\left[c(t)\left(z^{\prime}(t)\right)^{\alpha}\right]^{\prime}+q(t) f(x(t-\sigma))=0, t \geq T_{0} .
$$

So

$$
\left[c(t)\left(z^{\prime}(t)\right)^{\alpha}\right]^{\prime}=-q(t) f(x(t-\sigma)) \leq 0, t \geq T_{0} .
$$

It follows that the function $c(t)\left(z^{\prime}(t)\right)^{\alpha}$ is decreasing and $z^{\prime}(t)$ is eventually of one sign. We claim that $z^{\prime}(t)>0$.Otherwise, if there exist $t_{1}>T_{0}$, such that $z^{\prime}\left(t_{1}\right) \leq 0$. By using conditions (3-5), there exist $T_{1}>t_{1}$, such that $z^{\prime}\left(T_{1}\right)<0$. We have

$$
z(t) \leq z\left(T_{1}\right)+c\left(T_{1}\right)^{\frac{1}{\alpha}} z^{\prime}\left(T_{1}\right) \int_{T_{1}}^{t} \frac{d \xi}{c(\xi)^{\frac{1}{\alpha}}},
$$

from condition (1), we have $\lim _{t \rightarrow \infty} z(t)=-\infty$. This is a contradiction. Hence $z^{\prime}(t)>0$

Lemma 4. (First mean value theorem of integrals) If $f(x)$ and $g(x)$ are integrate in $[a, b] . g(x)$ is the same number in $[a, b]$ and $f(x) \in C[a, b]$. Then there exist $\xi \in[a, b]$, such that

$$
\int_{a}^{b} f(x) g(x) d x=f(\xi) \int_{a}^{b} g(x) d x .
$$

Lemma5. (Second mean value theorem of integrals) If $f(x)$ and $g(x)$ are integrate in $[a, b] . \quad f(x) \geq 0$ and is monotone decreasing in $[a, b]$. Then there exist $\varepsilon \in[a, b]$, such that

$$
\int_{a}^{b} f(x) g(x) d x=f(a) \int_{a}^{\varepsilon} g(x) d x \text {. }
$$

\section{Main Results}

The main result of this paper is the following. Theorem. Let

$$
D=\left\{(t, s) \mid t \geq s \geq t_{0}\right\}, H \in C(D, R),
$$

such that

(a) $H(t, s) \geq 0, t \geq s \geq t_{0} ; H(t, s)>0, t>s \geq t_{0}$;

(b) $\frac{\partial H}{\partial s} \leq 0$ in $\mathrm{D}$.

Suppose $f(x)$ such that

$$
\begin{aligned}
& \text { A1 } \int_{\eta}^{\infty} \frac{d u}{f(u)}<\infty, \int_{-\eta}^{-\infty} \frac{d u}{f(u)}<\infty, \eta>0 ; \\
& \text { A2 } f^{\prime}(x) \geq 0, x \neq 0 ; \\
& \text { A3 }-f(x y) \geq f(x y) \geq k f(x) f(y), x y>0, k \in N .
\end{aligned}
$$

If exist a function $\rho:\left[t_{0}, \infty\right] \rightarrow(0, \infty)$ such that $\rho^{\prime}(t) \geq 0$, and $\left[c(t-\sigma) \rho^{\prime}(t)\right]^{\prime} \leq 0, t \geq t_{0}+\sigma$.

If

$$
\limsup _{t \rightarrow \infty} \frac{1}{H\left(t, t_{0}\right)} \int_{t_{0}}^{t} H(t, s) \rho(s) q(s) f(1-p(s-\sigma)) d s=\infty
$$

then Eq. (1) is oscillatory.

Proof. Suppose to the contrary that Eq. (1) has a non-oscillatory solution $x(t)$. Without loss of generality we may assume that $x(t)$ is a eventually positive solution, then there exist $T_{0} \geq t_{0}+\tau+\sigma$, such that

$$
x(t)>0, x(t-\tau)>0, x(t-\tau-\sigma)>0, t \geq T_{0} .
$$

From Eq. (1) and Lemma 3, we have

$$
\left[c(t)\left(z^{\prime}(t)\right)^{\alpha}\right]^{\prime}+q(t) f(z(t-\sigma)-p(t-\sigma) x(t-\tau-\sigma))=0, t \geq T_{0} .
$$

By using $\mathrm{A} 2$, and $z(t)>x(t)$, then 


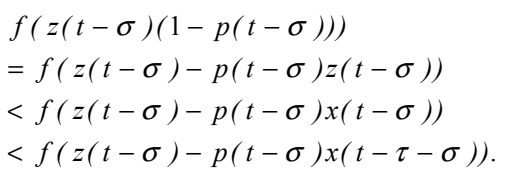

Furthermore, by using A3, we obtain

$$
f(z(t-\sigma)(1-p(t-\sigma))) \geq k f(z(t-\sigma)) f(1-p(t-\sigma)) .
$$

From (4) and (5), the following inequality is obtained.

$$
\begin{aligned}
& {\left[c(t)\left(z^{\prime}(t)\right)^{\alpha}\right]^{\prime}+q(t) f(z(t-\sigma)(1-p(t-\sigma)))} \\
& \leq\left[c(t)\left(z^{\prime}(t)\right)^{\alpha}\right]^{\prime}+q(t) f(z(t-\sigma)-p(t-\sigma) x(t-\tau-\sigma)) \\
& =0 .
\end{aligned}
$$

By using (6) and (7), we get

$$
\begin{aligned}
& {\left[c(t)\left(z^{\prime}(t)\right)^{\alpha}\right]^{\prime}+k q(t) f(1-p(t-\sigma)) f(z(t-\sigma))} \\
& \leq\left[c(t)\left(z^{\prime}(t)\right)^{\alpha}\right]^{\prime}+q(t) f(z(t-\sigma)(1-p(t-\sigma))) \\
& \leq 0 .
\end{aligned}
$$

In summary, we have

$$
\left[c(t)\left(z^{\prime}(t)\right)^{\alpha}\right]^{\prime}+k q(t) f(1-p(t-\sigma)) f(z(t-\sigma)) \leq 0, t \geq T_{0} .
$$

Let

$$
w(t)=\rho(t) \frac{c(t)\left(z^{\prime}(t)\right)^{\alpha}}{f(z(t-\sigma))}
$$

Clearly, $w(t)>0$. The first derivative of $t$ with respect to $w$ is obtained as follows.

$$
\begin{aligned}
w^{\prime}(t) & =\rho^{\prime}(t) \frac{c(t)\left(z^{\prime}(t)\right)^{\alpha}}{f(z(t-\sigma))}+\rho(t) \frac{\left(c(t)\left(z^{\prime}(t)\right)^{\alpha}\right)^{\prime}}{f(z(t-\sigma))} \\
& -\rho(t) \frac{c(t)\left(z^{\prime}(t)\right)^{\alpha}}{f^{2}(z(t-\sigma))} f^{\prime}(z(t-\sigma)) z^{\prime}(t-\sigma), t \geq T_{0} .
\end{aligned}
$$

We can easily get from (8)

$$
\left(c(t)\left(z^{\prime}(t)\right)^{\alpha}\right)^{\prime} \leq-k q(t) f(1-p(t-\sigma)) f(z(t-\sigma)),
$$

then

$$
\begin{aligned}
& \rho(t) \frac{\left(c(t)\left(z^{\prime}(t)\right)^{\alpha}\right)^{\prime}}{f(z(t-\sigma))} \leq \rho(t) \frac{-k q(t) f(1-p(t-\sigma)) f(z(t-\sigma))}{f(z(t-\sigma))} \\
& =-k \rho(t) q(t) f(1-p(t-\sigma)) \\
& \leq 0,
\end{aligned}
$$

so (9) is transformed into the following inequality.

$$
\begin{aligned}
w^{\prime}(t) \leq & -k \rho(t) q(t) f(1-p(t-\sigma))+\rho^{\prime}(t) \frac{c(t)\left(z^{\prime}(t)\right)^{\alpha}}{f(z(t-\sigma))} \\
& -\rho(t) \frac{c(t)\left(z^{\prime}(t)\right)^{\alpha}}{f^{2}(z(t-\sigma))} f^{\prime}(z(t-\sigma)) z^{\prime}(t-\sigma) .
\end{aligned}
$$

By Lemma 3, the function $c(t)\left(z^{\prime}(t)\right)^{\alpha}$ is not increase, then

$$
c(t)\left(z^{\prime}(t)\right)^{\alpha} \leq c(t-\sigma)\left(z^{\prime}(t-\sigma)\right)^{\alpha},
$$

Finally we obtained

$$
w^{\prime}(t) \leq-k \rho(t) q(t) f(1-p(t-\sigma))+\rho^{\prime}(t) \frac{c(t-\sigma)\left(z^{\prime}(t-\sigma)\right)^{\alpha}}{f(z(t-\sigma))} .
$$

So for all $t \geq T_{0}$, from (10), we have

$$
\begin{aligned}
& k \int_{T_{0}}^{t} H(t, s) \rho(s) q(s) f(1-p(s-\sigma)) d s \\
& \leq-\int_{T_{0}}^{t} H(t, s) w^{\prime}(s) d s+\int_{T_{0}}^{t} H(t, s) c(s-\sigma) \rho^{\prime}(s) \frac{\left(z^{\prime}(s-\sigma)\right)^{a}}{f(z(s-\sigma))} d s \\
& \leq-\left.H(t, s) w(s)\right|_{T_{0}} ^{t}+\int_{T_{0}}^{t} \frac{\partial H}{\partial s}(t, s) w(s) d s \\
& +H\left(t, T_{0}\right) \int_{T_{0}}^{t} c(s-\sigma) \rho^{\prime}(s) \frac{\left(z^{\prime}(s-\sigma)\right)^{a}}{f(z(s-\sigma))} d s \\
& \leq H\left(t, T_{0}\right)\left\{w\left(T_{0}\right)+\int_{T_{0}}^{t} c(s-\sigma) \rho^{\prime}(s) \frac{\left(z^{\prime}(s-\sigma)\right)^{a}}{f(z(s-\sigma))} d s\right\} .
\end{aligned}
$$

By Lemma 5, for $t \geq T_{0}$, there exist $\varepsilon \in\left[T_{0}, t\right]$ such that

$$
\begin{aligned}
& \int_{T_{0}}^{t} c(s-\sigma) \rho^{\prime}(s) \frac{\left(z^{\prime}(s-\sigma)\right)^{a}}{f(z(s-\sigma))} d s \\
& =c\left(T_{0}-\sigma\right) \rho^{\prime}\left(T_{0}\right) \int_{T_{0}}^{\varepsilon} \frac{\left(z^{\prime}(s-\sigma)\right)^{a}}{f(z(s-\sigma))} d s \\
& =c\left(T_{0}-\sigma\right) \rho^{\prime}\left(T_{0}\right) \int_{T_{0}}^{\varepsilon} \frac{\left(z^{\prime}(s-\sigma)\right)^{a-1}\left(z^{\prime}(s-\sigma)\right)}{f(z(s-\sigma))} d s,
\end{aligned}
$$

and by Lemma 4 and A1, the above formula can be transformed into

$$
\begin{aligned}
& c\left(T_{0}-\sigma\right) \rho^{\prime}\left(T_{0}\right) m \int_{T_{0}}^{\varepsilon} \frac{z^{\prime}(s-\sigma)}{f(z(s-\sigma))} d s \\
& =c\left(T_{0}-\sigma\right) \rho^{\prime}\left(T_{0}\right) m \int_{z\left(T_{0}-\sigma\right)}^{z(\varepsilon-\sigma)} \frac{d u}{f(u)} \\
& \leq c\left(T_{0}-\sigma\right) \rho^{\prime}\left(T_{0}\right) m \int_{z\left(T_{0}-\sigma\right)}^{\infty} \frac{d u}{f(u)}=M<\infty,
\end{aligned}
$$

where $\left(z^{\prime}(\xi-\sigma)\right)^{\alpha-1}=m, \xi \in\left[T_{0}, \mathcal{\varepsilon}\right] \subset\left[T_{0}, t\right]$.

Then

$$
k \int_{T_{0}}^{t} H(t, s) \rho(s) q(s) f(1-p(s-\sigma)) d s \leq H\left(t, T_{0}\right)\left\{w\left(T_{0}\right)+M\right\} .
$$

We have 


$$
\frac{1}{H\left(t, T_{0}\right)} \int_{T_{0}}^{t} H(t, s) \rho(s) q(s) f(1-p(s-\sigma)) d s \leq \frac{w\left(T_{0}\right)+M}{k} .
$$

Therefore,

$$
\limsup _{t \rightarrow \infty} \frac{1}{H\left(t, t_{0}\right)} \int_{t_{0}}^{t} H(t, s) \rho(s) q(s) f(1-p(s-\sigma)) d s<\infty .
$$

From (3), we have contradiction. The proof is complete.

\section{Conclusions}

In this paper, a new sufficient condition for a class of second order neutral delay differential equations is obtained and the corresponding result what was already obtained is generalized by this result. If $\alpha=1$, the equation

$$
\left[c(t)(x(t)+p(t) x(t-\tau))^{\prime}\right]^{\prime}+q(t) f(x(t-\sigma))=0, t \geq t_{0}
$$

is studied in [9]. The conclusion of theorem 1 of [9] is contained, so the result in this paper is more extensive. On the other hand, by using first and second mean value theorem of integrals, the proof of theorem is different from the proof in [4], so here is innovation of this paper.

\section{Acknowledgements}

The author would like to thank the referees for careful reading and useful suggestions improving this paper. This work is supported by the National Natural Science Foundation of China (Nos.11101251, 11402139 and 11471197) and Research Project Supported by Shanxi Scholarship Council of China (No. 2013-019).

\section{References}

[1] R.K. Zhang and W.T. Li, "Interval oscillation criteria for second order neutral nonlinear differential equations," Applied Mathematics and Computation, vol. 157, 2004, pp. 39-51.

[2] Q. Men and J.R. Yan, "Bounded oscillation for second order non-linear neutral delay differential equations in critical and non-critical case," Nonlinear Analysis, vol. 64, 2006, pp. 1543-1561.

[3] X.Y. Lin, "Oscillation of second-order nonlinear neutral differential equations," Journal of Mathematical Analysis and Applications, vol. 309, 2005, pp. 442-454.

[4] J.C. Jiang and X.P. Li, "Oscillation of second order nonlinear neutral differential equations," Applied Mathematics and Computation, vol. 135, 2003, pp. 531-540.

[5] M.M.A. ElSheikh and R. Sallam, "Oscillation criteria for second order functional differential equations," Applied Mathematics and Computation, vol. 115, 2000, pp. 113-121.

[6] Y. Sahiner, "On oscillation of second order neutral type delay differential equations," Applied Mathematics and Computation, vol. 150, 2004, pp. 697-706.

[7] S. James and W. Wong, "Necessary and sufficient conditions for oscillation of second order neutral differential equations," Journal of Mathematical Analysis and Applications, vol.252, 2004, pp. 342-352.

[8] Z.G. Ouyang, "Necessary and sufficient conditions for oscillation of odd order neutral delay parabolic differential equations," Applied Mathematics and Computation, vol. 16, 2003, pp. 1039-1045.

[9] H.J. Li and W.L. Liu, "Oscillation of second order neutral differential equations," Mathematics Computation Modelling, vol. 1, 1995, pp. 45-53.

[10] Y.X. Shi, "Oscillation criteria for nth order nonlinear neutral differential equations," Applied Mathematics and Computation, vol. 235, 2014, pp. 423-429. 